\title{
Compromiso neurológico en infección por COVID-19 en pacientes pediátricos
}

\section{Neurologic compromise in pediatric COVID-19 infection}

\author{
Vicente Quiroz S., ${ }^{\mathrm{a} b}$, Claudia Amarales O. ${ }^{\mathrm{a}, \mathrm{b}}$ \\ aUnidad de Neuropediatría Hospital Carlos van Buren. Valparaíso, Chile \\ bUniversidad de Valparaíso. Valparaíso, Chile
}

Recibido: 13 de mayo de 2020; Aceptado: 3 de junio de 2020

¿Qué se sabe del tema que trata este estudio?

La infección por COVID-19 genera compromiso principalmente pulmonar, sin embargo, existe un aumento de reportes de compromiso fuera del sistema respiratorio, que estarían mediados por la unión del virus a receptores de la enzima convertidora de angiotensina II (ACE2).
¿Qué aporta este estudio a lo ya conocido?

Es posible el compromiso a nivel del sistema nervioso en infecciones por COVID-19, tanto a nivel central (cefalea, compromiso de conciencia, patología cerebrovascular, etc.) como periférico (mialgias, anosmia, ageusia, entre otros), debiendo establecerse aún el mecanismo de este daño.

\section{Resumen}

La enfermedad por coronavirus ha extendido su compromiso más allá del sistema respiratorio con reportes crecientes de compromiso en diferentes sistemas, uno de ellos, el Sistema Nervioso. El potencial neuroinvasivo de este agente patógeno se explicaría por su neurotropismo dada la presencia de receptores de ACE2 a nivel de encéfalo y médula espinal, además del importante compromiso inflamatorio sistémico. El compromiso neurológico debido a la infección se ha dividido en Sistema Nervioso Central, destacando síntomas inespecíficos y leves como mareos y cefalea, así como cuadros graves con encefalitis y patología cerebrovascular, y Sistema Nervioso Periférico en donde la mayor relevancia guarda relación con la anosmia, ageusia y miositis. A nivel pediátrico el compromiso parece ser menor que en adultos, pero existe un reporte creciente en la literatura respecto a estos hallazgos. Es de gran importancia de contar con un adecuado registro y anamnesis que permita identificar precozmente el compromiso neurológico.
Palabras clave:

COVID-19;

SARS-CoV-2;

Neurotropismo;

Encefalitis;

ACE2

Correspondencia:

Vicente Quiroz S.

vicente.quiroz@postgrado.uv.cl 


\begin{abstract}
Coronavirus disease has extended its involvement beyond the respiratory system, with increasing reports of involving different systems, such as Nervous System. The neuroinvasive potential of this pathogen would be explained by its neurotropism given the presence of ACE2 receptors in the brain and spinal cord, in addition to the important systemic inflammatory involvement. The neurological involvement due to infection is divided between the central nervous system, highlighting non-specific and mild symptoms such as dizziness and headache, as well as severe symptoms with encephalitis and cerebrovascular pathology, and the peripheral nervous system, which mainly presents anosmia, ageusia, and myositis. Clinical symptomatology in pediatric patients seems to be less than in adults, but there is a growing report in the literature regarding these findings. Therefore, it is very important to have an adequate registry and anamnesis that allow early identification of neurological involvement.
\end{abstract}

Keywords:

COVID-19;

SARS-CoV-2;

Neurotropism;

Encephalitis;

ACE2

\section{Introducción}

La enfermedad por coronavirus SARS-CoV-2 (COVID-19) se reportó por primera vez en Wuhan, China, en el mes de diciembre de 2019 ${ }^{1}$. Desde ese momento ha existido múltiples reportes acerca de compromiso no sólo respiratorio, sino también de otros sistemas². La presente revisión tiene como objetivo evaluar el potencial neuroinvasivo del virus, describir los síntomas neurológicos descritos, su presencia en población pediátrica y la necesidad de una vigilancia estricta frente a nuevo compromiso neurológico que podría reportarse a futuro.

\section{Metodología}

Se realizó una búsqueda de la literatura en Medline/PubMed, SCOPUS, Scielo y EMBASE para los términos "coronavirus", "COVID-19", "SARS-CoV-2", "brain", "central nervous system", "nervous system" y "neurological" en diferentes combinaciones mediante el uso de operadores booleanos. Además, se revisaron las referencias de cada uno de los artículos seleccionados en busca de mayor evidencia.

\section{Potencial neuroinvasivo del SARS-CoV-2}

La mayoría de los coronavirus comparten tanto estructura como vía de infección, por lo que se plantea que los mecanismos de infección previamente descritos para otros virus son aplicables para SARS$\mathrm{CoV}-2$, entre ellos el neurotropismo ${ }^{3,4}$. Una partícula de coronavirus presenta cuatro proteínas estructurales denominadas proteína S (glucoproteína de espiga), proteína $\mathrm{E}$ (glucoproteína de envoltura), proteína $\mathrm{M}$ (glucoproteína de membrana) y proteína $\mathrm{N}$ (proteína de la nucleocápside), la primera de ellas es clave en la patogenia de la infección dado que puede reconocer y unirse a receptores en la superficie de células del ser humano, específicamente receptores de la enzima convertidora de angiotensina tipo II (ACE2) invadiendo las células humanas ${ }^{5,6}$. Existe evidencia que el encéfalo y médula espinal expresan receptores de ACE2 a nivel de células gliales y neuronas, lo que puede determinar que el tejido nervioso sea un potencial blanco de este patógeno ${ }^{7,8}$.

Sin embargo, no existe acuerdo respecto a si la vía acceso del virus al encéfalo es linfática, hematógena o axonal retrógrada (por ejemplo, a través de la infección de terminales nerviosos sensitivos o motores con transporte neuronal retrógrado o anterógrado) $)^{3,7,9}$. Existen autores que plantean que la vía axonal retrógrada y compromiso neurológico podrían incidir en la dificultad respiratoria reportada en la infección, esto en base a que otras cepas de coronavirus han demostrado infectar el tronco encefálico, lugar donde se encuentran los centros respiratorios (núcleo del tracto solitario y núcleo ambiguo) y por ende SARS-CoV-2 tendría este mismo potencial ${ }^{3}$. Esto, sumado al rol de la barrera hemato-encefálica en la capacidad de contener el virus y evitar que este acceda al encéfalo harían que la vía hematógena o linfática sea poco probable. No obstante, frente a un contexto de inflamación sistémica podría existir una disrupción de la barrera hematoencefálica y un ataque directo del virus a las células capilares endoteliales $^{7}$, facilitando su entrada al Sistema Nervioso Central (SNC).

Es importante determinar con futuras investigaciones si el compromiso neurológico reportado hasta el momento en las series corresponde a una infección directa del virus con demostración de la presencia del virus en líquido céfalo raquídeo (LCR ${ }^{10,11} \mathrm{o}$ al efecto del proceso inflamatorio sistémico provocado por la infección respiratoria. Por ahora, parece más plausi- 
ble lo segundo, es decir, que el daño cerebral sea producto de la disrupción de la homeostasis causada por compromiso pulmonar, renal, cardíaco y circulatorio ${ }^{7}$ que lleva a un daño de la barrera hematoencefálica e hipoxia cerebral con aumento del metabolismo anaerobio ${ }^{9}$.

\section{Síntomas neurológicos}

\section{Generalidades}

El compromiso neurológico reportado en la literatura corresponde en su mayoría reportes de casos, con variación porcentual según la serie y la definición de sintomatología neurológica, existiendo claridad que parece ser más frecuente en los pacientes con COVID-19 más grave 4 . El primer estudio que reporta el compromiso neurológico y permite la categorización de este corresponde a 214 personas hospitalizadas cursando infección por COVID-19 en Wuhan que describe que 78 pacientes $(36,4 \%)$ presentaron síntomas neurológicos, agrupándose en tres áreas: compromiso del SNC (53 pacientes, 24,8\%), del Sistema Nervioso Periférico (SNP) (19 pacientes, 8,9\%) y síntomas musculares (23 pacientes, 10,7\%), destacando que los síntomas neurológicos se hacen más frecuentes mientras más severo sea el estado clínico de la persona ${ }^{12}$. Un segundo reporte corresponde a una carta remitida por médicos del Hospital Universitario de Strasbourg en Francia quienes dan cuenta del compromiso neurológico de 58 pacientes ingresados a unidad de cuidados intensivos en quienes destaca la agitación psicomotora (39 pacientes), signos piramidales difusos (39 pacientes) caracterizados por aumento de reflejos osteotendíneos, clonus y reflejo cutáneo plantar extensor, la presencia de realce leptomeníngeo en resonancia magnética de encéfalo en 8 pacientes y secuelas posteriores con alteración de funciones ejecutivas en 15 personas (atención, desorientación o incapacidad de organizar movimientos en respuesta a un comando ${ }^{13}$.

\section{Síntomas de compromiso del Sistema Nervioso Central (SNC)}

Los síntomas asociados al SNC son variados, ocurriendo con mayor frecuencia la presencia de cefalea y mareos, pero pueden llegar a ser tan graves como parálisis flácida con mielitis, compromiso de conciencia, enfermedad cerebrovascular o encefalopatía necrotizante hemorrágica aguda ${ }^{9,14-16}$.

En el caso de la enfermedad cerebrovascular se ha planteado que los eventos isquémicos se encuentran asociados a una reacción inflamatoria sistémica y estado protrombótico desencadenado por el compro- miso fuera del SNC. Se ha descrito un aumento de riesgo particularmente en pacientes jóvenes, debiendo investigarse aún las causas tras ello ${ }^{17,18}$. En el caso del aumento de riesgo de accidente cerebrovascular hemorrágico este sería secundario a fluctuaciones de la presión arterial por compromiso de receptores de ACE2 $2^{9,16}$.

El compromiso de conciencia ha sido reportado principalmente en adultos mayores con infecciones graves, describiendo electroencefalogramas con hallazgos inespecíficos como lentitudes difusas y focales tanto en áreas frontales como temporales ${ }^{13,19}$.

El primer caso de encefalitis se presentó en un hombre de 24 años que cursó con un estado epiléptico refractario, cuyo estudio de LCR mostró una elevación de leucocitos y presencia de material genético de COVID-19 por PCR con una resonancia magnética que mostró una hiperintensidad a nivel del lóbulo temporal mesial derecho y del hipocampo, pudiendo estos hallazgos ser secundarios tanto a cambios postconvulsivos como a una encefalopatía $\operatorname{aguda}^{11}$.

\section{Síntomas de compromiso del Sistema Nervioso Periférico (SNP)}

El principal compromiso reportado corresponde a la anosmia y ageusia, con porcentajes variables en las series desde un $5,1 \%$ y $5,6 \%$ respectivamente ${ }^{12}$, lo que llevó a la Sociedad Española de Neurología el 21 de marzo de 2020 plantear como plausible la relación del aumento de número de reportes de pérdida de olfato y la actual pandemia por COVID-19. Posteriormente se definió la anosmia aguda como uno de los síntomas de infección por SARS-CoV-2 y la anosmia aguda aislada (sin otros síntomas virales, sin mucosidad y sin otra causa) como un posible síntoma atribuible a COVID-1920. El 27 de abril de 2020, el Ministerio de Salud de Chile incluyo en su definición de caso sospechoso la anosmia o ageusia aguda ${ }^{21}$. Es importante destacar que la incidencia de esta sintomatología puede estar subreportada dado que la alteración del gusto y/o olfato no suele preguntarse en la anamnesis inicial ${ }^{22}$. En el caso de pacientes pediátricos, esto puede ser aún más difícil de objetivar.

En tercer lugar de frecuencia se encontraría el daño del músculo esquelético manifestado clínicamente como una miositis, que se ha definido como la presencia de mialgias asociadas a una elevación de la creatina quinasa $(\mathrm{CK})>200 \mathrm{IU} / \mathrm{l}$ y que sería secundario a la presencia de ACE2 a nivel de músculo esquelético ${ }^{12,16,23}$.

Existen además reportes aislados de compromiso de la visión, síndrome de Guillain-Barré, neuralgia y plexopatía $^{24-27}$. 


\section{Compromiso neurológico en pediatría}

La infección por COVID-19 en población pediátrica, en comparación a los adultos, ha mostrado una menor prevalencia y en aquellos casos positivos, menor severidad de síntomas como fiebre, tos o disnea, encontrándose en discusión si esto se debe a menor expresión de receptores ACE2 e inmadurez de estos (lo que haría menos afín la unión de él virus a él), un sistema inmunológico en formación o menor exposición debido a medidas ambientales como la suspensión de las actividades escolares presenciales ${ }^{28-31}$. Es importante señalar que cohorte observacional de 36 pacientes pediátricos en Zhejiang, China reportó 10 casos que se presentaron con cefalea ${ }^{28}$.

El primer caso clínico publicado de encefalitis asociada a COVID-19 fue reportado en un niño de 11 años ingresado por un estado epiléptico refractario cuyo análisis de LCR mostró un aumento de leucocitos, con proteínas y glucosa en rango normal con demostración del virus tanto en nasofaringe como $\mathrm{LCR}^{32}$.

El segundo caso corresponde a una lactante de 6 semanas que consultó por un cuadro de un día de evolución de tos, fiebre y episodios breves (aproximadamente 10 segundos) de desconexión del medio, desviación de la mirada sostenida hacia arriba y aumento del tono de ambas extremidades inferiores, con un examen neurológico entre eventos sin alteraciones. Se realizó estudio con PCR en vía respiratoria resultando positivo para SARS-CoV-2 y Rhinovirus/Enterovirus. El estudio de LCR resultó normal y no se pudo realizar búsqueda de COVID-19 en LCR. El electroencefalograma mostró un exceso de transientes agudos temporales para la edad y una lentitud intermitente delta a nivel del vertex. Los autores interpretaron que se trató de una infección por dos agentes virales que causó el cuadro clínico ${ }^{33}$.

\section{Síntomas neurológicos posibles}

En los últimos años múltiples síndromes neurológicos asociados a otros coronavirus no COVID-19, han sido reportados en la literatura, tales como meningitis viral, encefalomielitis diseminada aguda postinfecciosa, encefalitis de tronco post infecciosa o convulsiones febriles $^{34,35}$ por lo que una adecuada vigilancia de pacientes que cursan o cursaron una infección por coronavirus y posteriormente presentan algún síntoma neurológico es de vital importancia para la adecuada caracterización del compromiso a nivel del Sistema Nervioso.

\section{Conclusiones y proyecciones}

La pandemia por COVID-19 ha significado una reestructuración del trabajo de múltiples especialidades médicas, entre ellas la Neurología Pediátrica, puesto que una infección que en primera instancia parecía ser netamente respiratoria, ha probado comprometer múltiples órganos y sistemas. Es relevante entender que las manifestaciones neurológicas descritas hasta ahora asociadas al SARS-CoV-2 son por ahora obtenidas de reportes clínicos aislados y estudios de baja calidad metodológica. Es esperable que, con el paso de las semanas, se acumule nueva evidencia de manifestaciones clínicas, que puedan cambiar conocimiento actual.

Actualmente es posible señalar que el potencial neuroinvasivo sería principalmente secundario a la tormenta inflamatoria debido al compromiso sistémico, requiriendo mayor investigación sobre el rol directo del virus a nivel del Sistema Nervioso, tanto a nivel central, como periférico.

A nivel internacional se han implementado protocolos para el manejo de la enfermedad por COVID-19 por neurólogos en los que destaca la necesidad del reconocimiento precoz de complicaciones neurológicas en pacientes gravemente enfermos que podrían resultar en secuelas a largo plazo o dificultad en el manejo de la condición, esto pese a su relativa infrecuencia ${ }^{16}$. Iniciativas como las de la Sociedad Española de Neurología con registro de manifestaciones y complicaciones neurológicas de actualización semanal ${ }^{25}$ aparecen como una alternativa interesante de replicar a nivel local, además de permitir a países de América Latina que se encuentran en etapas más tempranas de la pandemia el hacer un análisis más apropiado de los datos y determinar si las manifestaciones neurológicas observadas son directas por el virus, indirectas por el estado inflamatorio o ambas. Los profesionales que atienden a población pediátrica deben reconocer síntomas inespecíficos en etapas tempranas y que requieren una anamnesis dirigida y adaptada en menores de edad tales como la cefalea, anosmia o dolor muscular. A futuro existen múltiples desafíos donde, además de lo anteriormente mencionado, deberán considerarse nuevas perspectivas como lo son el cuidado de niños, niñas y adolescentes con comorbilidad neurológica en contexto de pandemia o los efectos indirectos del virus en el neurodesarrollo y salud mental.

\section{Conflicto de intereses}

Los autores declaran no tener conflicto de intereses. 


\section{Referencias}

1. Zhu N, Zhang D, Wang W, et al. A novel coronavirus from patients with pneumonia in China, 2019. N Engl J Med. 2020;382(8):727-33.

2. Wang D, Hu B, Hu C, et al. Clinical Characteristics of 138 Hospitalized Patients with 2019 Novel CoronavirusInfected Pneumonia in Wuhan, China. JAMA - J Am Med Assoc. 2020;323(11):1061-9.

3. Li YC, Bai WZ, Hashikawa T. The neuroinvasive potential of SARS-CoV2 may be at least partially responsible for the respiratory failure of COVID-19 patients. J Med Virol. 2020;24-7.

4. Asadi-Pooya AA, Simani L. Central nervous system manifestations of COVID-19: A systematic review. Vol. 413, Journal of the Neurological Sciences. Elsevier B.V.; 2020. https://doi. org/10.1016/j.jns.2020.116832.

5. Su S, Wong G, Shi W, et al. Epidemiology, Genetic Recombination, and Pathogenesis of Coronaviruses. Vol. 24, Trends in Microbiology. Elsevier Ltd; 2016. p. 490502.

6. Zhao Y, Zhao Z, Wang Y, Zhou Y, Ma Y, Zuo W. Single-cell RNA expression profiling of ACE2, the putative receptor of Wuhan 2019-nCov. bioRxiv [Internet]. 2020;2020.01.26.919985. Disponible en: https://www.biorxiv.org/content/10.1101/ 2020.01.26.919985v1.

7. Baig AM, Khaleeq A, Ali U, Syeda H. Evidence of the COVID-19 Virus Targeting the CNS: Tissue Distribution, Host-Virus Interaction, and Proposed Neurotropic Mechanisms. ACS Chem Neurosci. 2020;0-3.

8. Nemoto W, Yamagata R, Nakagawasai $\mathrm{O}$, et al. Effect of spinal angiotensinconverting enzyme 2 activation on the formalin-induced nociceptive response in mice. Eur J Pharmacol [Internet]. 2020;872:172950. Disponible en: https:// doi.org/10.1016/j.ejphar.2020.172950.

9. Wu Y, Xu X, Chen Z, et al. Nervous system involvement after infection with COVID-19 and other coronaviruses. Brain, Behavior, and Immunity. Academic Press Inc.; 2020.

10. Xinhua. Beijing hospital confirms nervous system infections by novel coronavirus [Internet]. [citado el 3 de mayo de 2020]. Disponible en: http://www.xinhuanet. com/english/2020-03/05/c_138846529. htm.

11. Moriguchi T, Harii N, Goto J, et al. A first case of meningitis/encephalitis associated with SARS-Coronavirus-2. Int J Infect
Dis. 2020;94:55-8.

12. Mao L, Wang M, Chen S, et al. Neurological Manifestations of Hospitalized Patients with COVID-19 in Wuhan, China: A Retrospective Case Series Study. SSRN Electron J. 2020.

13. Helms J, Kremer S, Merdji H, et al. Neurologic Features in Severe SARSCoV-2 Infection. N Engl J Med. 2020.

14. Poyiadji N, Shahin G, Noujaim D, Stone M, Patel S, Griffith B. COVID19-associated Acute Hemorrhagic Necrotizing Encephalopathy: CT and MRI Features. Radiology. 2019;2(Cdc):1-11.

15. Zhao K, Huang J, Dai D, Feng Y, Liu L, Nie S. Acute myelitis after SARS-CoV-2 infection: a case report. medRxiv. 2020.

16. Jin $\mathrm{H}$, Hong $\mathrm{C}$, Chen $\mathrm{S}$, et al. Consensus for prevention and management of coronavirus disease 2019 (COVID-19) for neurologists. Stroke Vasc Neurol. 2020;2019(M):1-6.

17. Oxley TJ, Mocco J, Majidi S. LargeVessel Stroke as a Presenting Feature of Covid-19 in the Young. N Engl J Med [Internet]. 2020;382(20):e60(1)-e60(3). Available from: https://www.nejm.org/ doi/full/10.1056/NEJMc2009787?url_ ver $=$ Z39.88-2003\&rfr_id=ori:rid:crossref. org\&rfr_dat $=$ cr_pub 0pubmed

18. Valderrama EV, Humbert K, Lord A, Frontera J, Yaghi S. Severe Acute Respiratory Syndrome Coronavirus 2 Infection and Ischemic Stroke. Stroke [Internet]. 2020;STROKEAHA120030153. Disponible en: http://www.ncbi.nlm.nih. gov/pubmed/32396456.

19. Filatov A, Sharma P, Hindi F, Espinosa PS. Neurological Complications of Coronavirus Disease (COVID-19): Encephalopathy. Cureus. 2020;12(3).

20. (SEN) SE de N. Recomendaciones de la Sociedad Española de Neurología (SEN) en relación con la pérdida de olfato como posible síntoma precoz de infección por COVID-19 [Internet]. 2020 p. 1-2. Disponible en: http://www.sen.es/ noticias-y-actividades/222-noticias/covid19-informacion-para-pacientes/2663covid-recomendaciones-de-la-sociedadespanola-de-neurologia-sen-en-relacioncon-la-perdida-de-olfato-como-posiblesintoma-precoz-de-infeccion-porcovid-19.

21. Ministerio de Salud. Consejo Asesor del Ministerio de Salud amplía definición de casos sospechosos y confirmados de COVID-19 [Internet]. 2020 [cited 2020 Apr 27]. Disponible en: https://www. minsal.cl/consejo-asesor-del-ministeriode-salud-amplia-definicion-de-casossospechosos-y-confirmados-de-covid-19/
22. Vaira LA, Salzano G, Deiana G, De Riu G. Anosmia and Ageusia: Common Findings in COVID-19 Patients. Laryngoscope. 2020.

23. Cabello-Verrugio C, Morales MG, Rivera JC, Cabrera D, Simon F. ReninAngiotensin System: An Old Player with Novel Functions in Skeletal Muscle. Med Res Rev [Internet]. 2015;35(3):43763. Disponible en: http://doi.wiley. com/10.1002/med.21343.

24. Alberti P, Beretta S, Piatti M, et al. Guillain-Barré syndrome related to COVID-19 infection. Neurol Neuroimmunol neuroinflammation [Internet]. 2020;7(4):1-4. Disponible en: http://www.ncbi.nlm.nih.gov/ pubmed/32350026.

25. (SEN) SE de N. Registro de manifestaciones/complicaciones neurológicas en pacientes con infección por el virus SARS-CoV-2 [COVID-19] de la Sociedad Española de Neurología - 27 de abril de 2020 [Internet]. 2020 [citado el 3 de mayo de 2020]. Disponible en: http:// www.sen.es/index.php?option=com_cont ent\&view $=$ article\&id $=26598$ catid $=91$

26. Gutiérrez-Ortiz C, Méndez A, RodrigoRey S, et al. Miller Fisher Syndrome and polyneuritis cranialis in COVID-19. Neurology. 2020;241:10.1212/ WNL.0000000000009619.

27. Dinkin M, Gao V, Kahan J, et al. COVID-19 presenting with ophthalmoparesis from cranial nerve palsy. Neurology. 2020.

28. Qiu H, Wu J, Hong L, Luo Y, Song Q, Chen D. Clinical and epidemiological features of 36 children with coronavirus disease 2019 (COVID-19) in Zhejiang, China: an observational cohort study. Lancet Infect Dis. 2020.

29. Dong Y, Mo X, Hu Y, et al. Epidemiological Characteristics of 2143 Pediatric Patients With 2019 Coronavirus Disease in China. Pediatrics. 2020 Mar 16.

30. Lu X, Zhang L, Du H, et al. SARS-CoV-2 Infection in Children. N Engl J Med [Internet]. 2020 Apr 23;382(17):1663-5. Available from: http://www.nejm.org/ doi/10.1056/NEJMc2005073.

31. Ludvigsson JF. Systematic review of COVID-19 in children shows milder cases and a better prognosis than adults. Acta Paediatrica, International Journal of Paediatrics. Blackwell Publishing Ltd; 2020.

32. McAbee GN, Brosgol Y, Pavlakis S, Agha R, Gaffoor M. Encephalitis Associated with COVID-19 Infection in an 11 YearOld Child. Pediatr Neurol [Internet]. 2020 Apr; Disponible en: https:// 
linkinghub.elsevier.com/retrieve/pii/ S0887899420301430.

33. Dugue R, Cay-Martínez KC, Thakur KT, et al. Neurologic manifestations in an infant with COVID-19.

Neurology [Internet]. 2020;10.1212/
WNL.0000000000009653. Disponible en: https://doi.org/10.5061/dryad.v41ns1rsc.

34. Nath A. Neurologic complications of coronavirus infections. Neurology. 2020;10.1212/WNL.0000000000009455.

35. Carman KB, Calik M, Karal Y, et al. Viral etiological causes of febrile seizures for respiratory pathogens (EFES Study).

Hum Vaccines Immunother [Internet]. 2019;15(2):496-502. Disponible en https://doi.org/10.1080/21645515.2018.15 26588. 